\title{
A HIERARCHICAL ARCHITECTURE FOR AN ENERGY MANAGEMENT SYSTEM
}

\author{
Krzysztof Piotrowski, ${ }^{1, *}$, Augusto Casaca ${ }^{2}$, Marco E. T. Gerards ${ }^{3}$, Marijn Jongerden ${ }^{3}$, \\ Francisco Melo ${ }^{4}$, Daniel Garrido ${ }^{5}$, Marcel Geers ${ }^{6}$, Jacobo Peralta $^{7}$ \\ ${ }^{1}$ IHP, Frankfurt (Oder), Germany \\ ${ }^{2}$ INESC-ID/INOV, Lisboa, Portugal \\ ${ }^{3}$ University of Twente, Enschede, the Netherlands \\ ${ }^{4}$ EDP Distribuição, Lisboa, Portugal \\ ${ }^{5}$ Universidad de Malaga, Malaga, Spain \\ ${ }^{6}$ Alliander N.V., Arnhem, the Netherlands \\ ${ }^{7}$ Cemosa, Malaga, Spain \\ "piotrowski@ihp-ffo.de
}

Keywords: Control Systems, Energy Management, Renewable Energy Sources, Smart Grids

\begin{abstract}
This paper introduces an innovative energy management system architecture for Smart Grids, designed in the European 7 th framework program project e-balance. The architecture is hierarchical and fractal-like, which results in better scalability and reuse of algorithms and programming code for energy management. It supports both micro-grids and distributed energy resources. The system is structured into two main parts - the communication platform and the energy management platform. The former provides a common data exchange layer, while the latter provides the local energy management logic, currently supporting energy balancing and grid resilience services. The system is being deployed in two pilots: in Portugal and in the Netherlands, where use cases on energy balancing, grid monitoring and grid reliability will be demonstrated.
\end{abstract}

\section{Introduction}

The increasing penetration of renewables causes issues related to the mismatch between production and consumption in the temporal dimension. The electricity is sometimes discarded, e.g., by curtailment of PV panels, or transported over long distances, causing losses, requiring investments in the infrastructure and decreasing the lifetime of the equipment. An overview of these issues is given in [1].

Thus, it is especially important to allow balancing the energy consumption and production to match them at the local level. To address this issue in an efficient way, it is advantageous to have a scalable solution that can be applied at every level of the grid to provide local decisions and control. The support for islanded microgrids is a further advantage.

In this paper we propose a hierarchical, robust and flexible energy management system for the future electricity distribution grid that tackles these problems by allowing balancing the production and consumption of electricity and monitoring the state of the grid. The system is developed within the context of the European 7 th framework program project e-balance [2].

The general aim of the e-balance project is to design a management system, which increases the efficiency and the quality of the grid, while taking into consideration the interests of the end-users. Because the interaction with the end-users is crucial, the project includes not only technical aspects, but also social and economic ones. In this paper, we only focus on the technical aspects of the project, and more precisely on the architecture of the management system [3].

The system consists of two layers: the communication platform provides the means for secure and reliable data exchange between the system's devices, while the energy management platform provides the control logic based on the exchanged data. The flexibility of the approach is due to the fact that the data can be defined at will, allowing extending and adapting the energy management logic to all specific and future needs. And this task is simplified as the energy management platform is modular. It is defined as a set of services, each providing a distinct functionality.

A hierarchical and fractal-like architecture is chosen. As such, the energy management system, from now on called the e-balance system, considers a neighbourhood as an individual unit to be managed at a higher level; a large neighbourhood consists of smaller units, and so on. This is an innovative approach that allows using the same management logic at different levels of the electricity grid, like a fractal, while still supporting fine grained management. The major advantage is the application of similar control algorithms at different levels of the hierarchy, resulting in better scalability and reuse of algorithms and, possibly, of programming code. It also reflects the nature of the energy grid, at different levels, all of them showing a fractal-like pattern.

The e-balance system is being deployed in two pilots running in Portugal and in the Netherlands. A large set 
of features (more than twenty), defining the use cases on energy balancing, on grid monitoring and reliability, will be demonstrated. Many of these features involve end-users [4]. The balancing of production and consumption of energy and the improvement of the reliability of the grid are important items of research within the smart grid [5] and this justifies their relevance within e-balance.

This paper is organized as follows. Section 2 makes a short review of related work. Section 3 presents the ebalance system architecture, while Section 4 describes the atomic management unit. More details on the communication and the energy management platforms are presented in Section 5 and Section 6, respectively. Section 7 refers to the pilots and, finally, Section 8 concludes the paper.

\section{Related Work}

The CEN-CENELEC-ETSI Smart Grid Coordination Group defined the Smart Grid Architecture Model (SGAM) [6]. SGAM identifies five grid domains, five interoperability layers and six management zones. The e-balance system is in line with the SGAM, except that e-balance only considers the distribution grid, the distributed energy resources and the customer premises domains, leaving the bulk generation and transmission domains out of scope.

SmartC2Net [7], another 7th framework program running project, aims to find solutions for enabling smart grid operations on top of a heterogeneous communication infrastructure. It also proposes a system architecture that follows the standards, but the main difference from the e-balance architecture is the hierarchical and fractal-like approach used in e-balance.

C-DAX, which is also a 7th framework program project, introduces an integrated communication and information infrastructure based on the Information Content Networking concept [8]. The e-balance presents distinctive features, regarding not only the middleware solution, but also the hierarchical architecture.

The paper by Kim et al [9] focuses on an architecture that supports machine-to-machine communications for thousands of devices deployed within the grid. However, the paper focuses on a solution based on software-defined networks.

Compared to e-balance, the Monitor BT project has a similar communication network philosophy, yet, it features a distinctive approach at the middleware level and it lacks a hierarchical and fractal-like structure for the management infrastructure [10].

Compared to the other approaches e-balance goes much further beyond state of the art allowing very dynamic and complex set-ups with real-time energy balancing for future energy grids. Some of the features of e-balance cannot be used yet, due to legal or technical issues related to energy grids, but will be needed in the future to cope with the dynamic changes in the energy grid.

\section{System Architecture}

The e-balance system architecture follows the electricity grid's hierarchical structure, as is presented in Figure 1. In our approach we have adapted the SGAM architecture to match the objectives of e-balance by adding additional details about domains and components that are the focus of the architecture, while omitting the others that are out of scope. For easing the representation, the three-dimensional SGAM model was transformed to a two-dimensional hierarchical model, which is easier to handle. The Energy Grid level corresponds to the SGAM domains. The Market, Global Data Access and Operations layers correspond to the corresponding SGAM zones. The SGAM interoperability layers are distributed among the ebalance system components. The bulk generation and transmission levels are collapsed as they are out of the scope of the architecture. Within e-balance we also subdivide the Distribution level into two segments: Medium Voltage (MV) and Low Voltage (LV).

For clarification, in the remainder of this paper we use the term grid to refer to the energy grid. In contrast, the term network is used to represent a communication network within the e-balance system.

In Figure 1, the system components are represented by dark blue coloured shapes. The blue boxes represent the management units, the core of the e-balance system. The blue ovals represent the sensors and actuators that allow interaction with the grid. The light blue shapes represent the counterparts at the Bulk Generation and Transmission level, which are out of the scope of the presented solution.

The system architecture involves several classes of management units and this figure depicts the hierarchical tree of these with a single management unit level for each voltage level in the grid. In very dense grids, intermediate management unit levels may be applied to reduce the data load in the network and to further distribute the local decisions.

The yellow coloured boxes represent the grid and the devices within the grid. Finally, the red coloured boxes represent virtual layers such as, the Market, Global Data Access and Operations. Green shapes define the locations of the components.

The different lines in the figure represent different kinds of interaction between the components they connect. Black lines represent the communication network, i.e., the data exchange that involves the management units, the sensors and the actuators. Blue lines represent the interaction between the e-balance system and the grid, i.e., gathering data from sensors located in the grid and sending control signals that trigger actions by actuators in the grid. The orange lines represent the energy flows. 


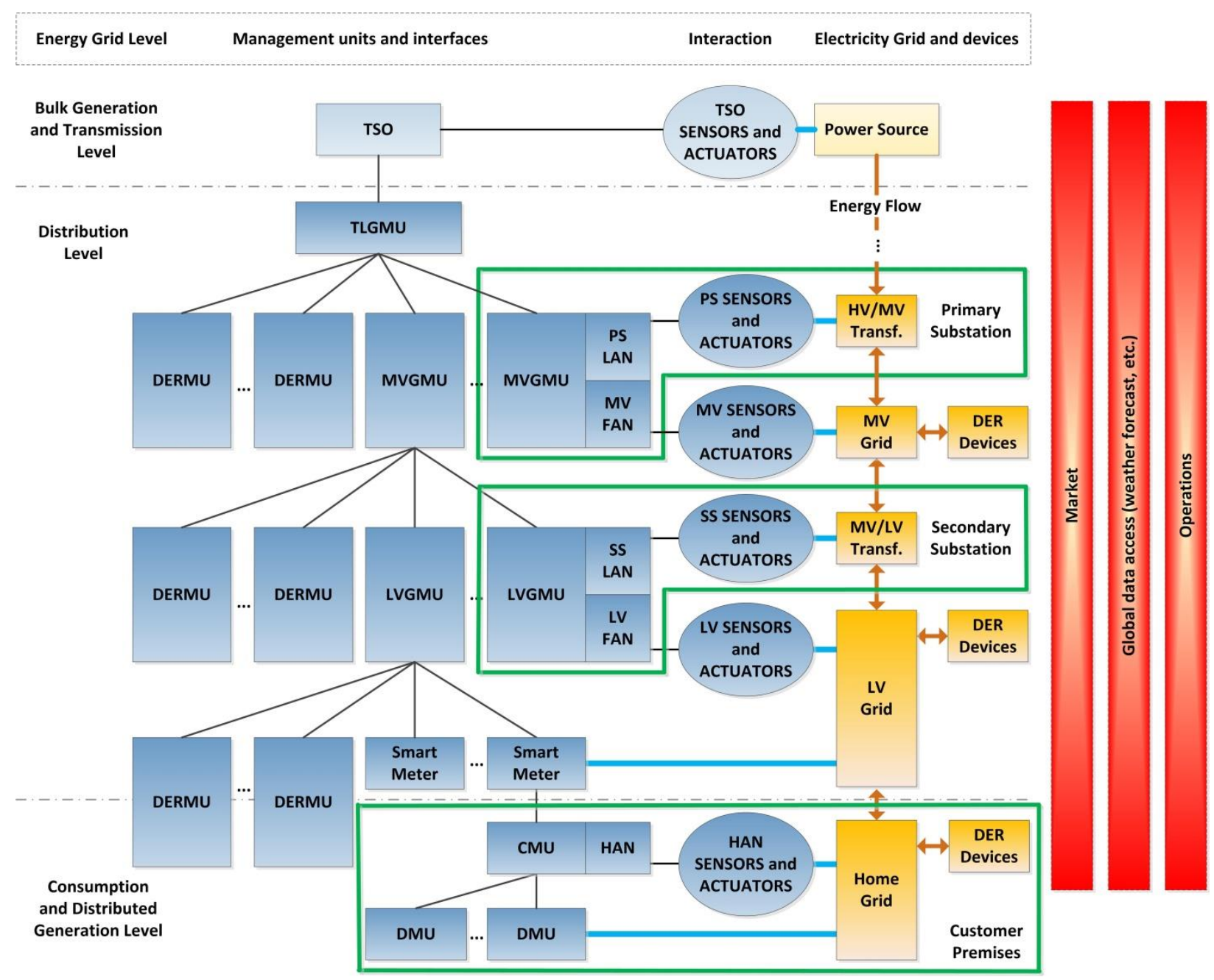

Figure 1 The e-balance system architecture

The management units have a common architecture, which is described in detail in the next section. Depending on the level, the management units may have different roles and duties. The processes executed on the management units may operate on behalf of different stakeholders and process data from other stakeholders. However, since the concept of data collection and processing is similar at every level, the management algorithms applied at different management levels share the same conceptual base, which improves the scalability of the approach.

\subsection{Device Management Unit (DMU)}

The device level is the lowest level represented in the architecture. A device may be of any kind, e.g., a home appliance that only consumes energy, an energy generation unit or a storage unit. The DMU is a device unit that controls the current state of all the components within the device. The DMU is equipped with a communication module or gateway that allows upward communication with the higher level management unit, i.e., the Customer Management Unit (CMU).

In order to support interoperability with all devices available on the market, as well as with devices to appear on the market in the future, it was decided to focus on standard solutions and not to develop a DMU on our own. This improves the e-balance coverage, scalability and applicability. This interoperability mainly involves the communication technologies to be used within the Home Area Network (HAN) that connects the DMUs and their corresponding CMU. The currently used technologies include Z-Wave, ZigBee and Bluetooth Low Energy. But the modular approach allows extending this set by new ones.

\subsection{Customer Management Unit (CMU)}

The CMU controls all the DMUs at the customer premises. The CMU is aware of the state of each device as well as of the individual and cumulative energy consumption and production figures, wherever applicable. Thus, it could also provide the accounting functionality of a domestic smart energy meter. However, in order to do this correctly the CMU has to be approved by a notified body. Thus, the Smart Meter is introduced as an additional layer in the architecture. But, this distinction can also be virtual, combining these two devices in one unit - the CMU. This provides the consumers with the flexibility of choice for the brand of the CMU, in case it is certified for energy accounting. This also allows separating the customer and the distribution grid domains regarding the data and device 
ownership, as well as to identify and highlight the interface between these two domains. Nonetheless, the e-balance architecture also comprises the integration of smart meters.

The CMU is equipped with several communication modules or gateways. It communicates with its underlying DMUs, and it also communicates with HAN sensors and actuators.

\subsection{Distributed Energy Resource Management Unit (DERMU)}

The DERMU is the device management unit for some specific DER devices that may be connected to different voltage level parts of the grid. The customer may give some of the control over the DER devices at her premises to external stakeholders, such as the Distribution System Operator (DSO) or an aggregator, according to the regulatory framework. The difference between the DMU and the DERMU is that the latter can directly communicate with a higher level management unit in the distribution domain, while the former needs the CMU for that. A DERMU attached to or integrated in a PV inverter might, for example, be directly controlled by the Low Voltage Grid Management Unit (LVGMU) or by another external source, but it might also communicate with the CMU, allowing for a different kind of control.

\subsection{Low Voltage Grid Management Unit (LVGMU)}

The management level above the CMUs consists of LVGMUs. These management units are located at the secondary substations and each of them controls the sensors and the actuators, as well as CMUs and DERMUs located in the area of the grid, supplied with energy by this secondary substation. A LVGMU is equipped with communication gateways to communicate with the upper and lower network layers within the e-balance management hierarchy. It is also equipped with communication gateways for providing communications with sensors and actuators located at the MV/LV transformer (Secondary Substation Local Area Network - SS-LAN) and also at the LV grid feeders related to the secondary substation (Low Voltage Field Area Network - LV-FAN). All these communication gateways may be different, depending on the technologies used in each part of the network.

\subsection{Medium Voltage Grid Management Unit $(M V G M U)$}

An MVGMU is similar to its counterpart for the LV part of the grid. However, it resides at a primary substation. It is equipped with upward and downward communication gateways and controls all the sensors/actuators in the MV grid. It also interacts with the LVGMUs located at secondary substations related to this primary substation and the DERMUs related to the MV grid connected DER, placed in this area. In order to interact with the sensors and actuators at the HV/MV transformer, the MVGMU is equipped with a Primary
Substation Local Area Network (PS-LAN) gateway. Similar, for communicating with the sensors and actuators in the MV grid related to the primary substation, a Medium Voltage Field Area Network (MV-FAN) gateway is available at the MV grid management unit. Again, the communication gateways may use different communication technologies.

\subsection{Top Level Grid Management Unit (TLGMU)}

Finally, the TLGMU controls all the MVGMUs as well as all the DERMUs for DERs connected directly to the MV grid, i.e., it collects all the status data and sends control signals to all the lower level management units. The TLGMU may be considered as a control center that provides also interfaces for management tools, like a supervisory control and data acquisition (SCADA) system, Outage Management System, Distribution Management System (DMS) and Metering Data Management (MDM). The TLGMU communicates with higher level management units held above the distribution domain, for instance, with the Transmission Service Operator (TSO).

This hierarchical approach can reach even further, creating higher levels in the hierarchy with management units that provide bulk balancing for a large region. This is the strength of this approach and it provides scalability for the energy balancing that eventually needs to be done in the grid.

\section{The Management Unit}

A management unit controls all its direct subordinate system elements, i.e., lower level management units, sensors and actuators. It takes control decisions based on the user configuration and interaction, but also on the context consisting of data received from its parent management unit and the data obtained from the subordinate system elements.

The core functionality of the management unit that interacts with the respective part of the energy infrastructure is split into two main blocks: the Communication Platform and the Energy Management Platform. The former is responsible for data gathering and exchange within the network, while the latter represents the logic that takes the local decisions based on the grid data. This logic is realized as a set of services, each providing a given functionality. The architecture of the management unit is presented in Figure 2. This figure provides the general view on the main functional blocks that will be described further in detail.

The management unit communicates with sensors and actuators that interact directly with the segment grid the unit is responsible for. It communicates also with its subordinate units. Finally, each management unit, except the TLGMU, also communicates with its parent management unit. The TLGMU instead, communicates with the TSO (embedding all upstream grid master components). All these mentioned communications may 


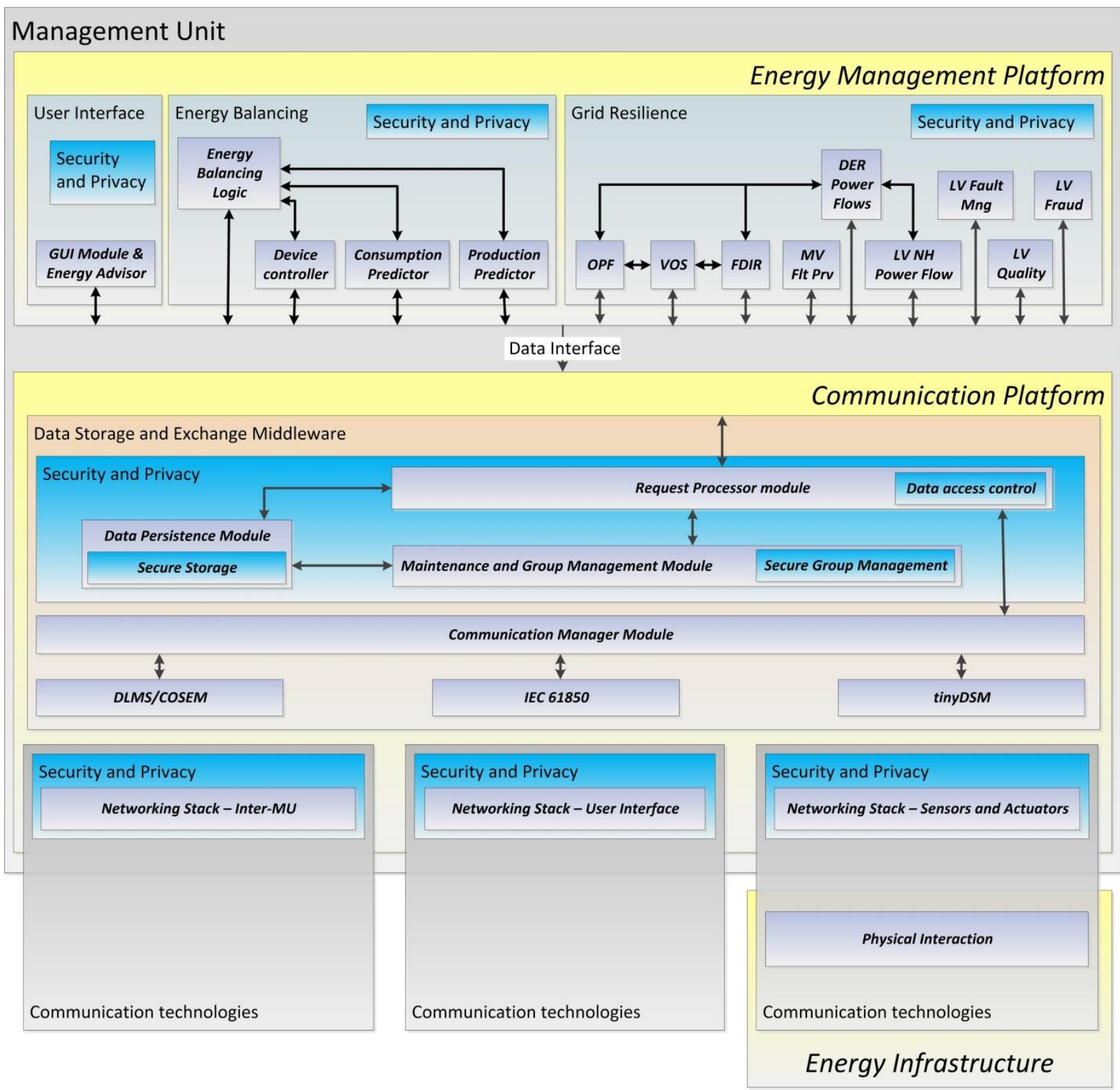

Figure 2 Architecture of the e-balance management unit

use different communication technologies and thus, they may require different networking stacks. Thus, the architecture shown in Figure 2, allows for several networking stacks, each for a different purpose.

The Data Storage and Exchange Middleware is placed on top of the networking stacks. It provides the abstract and common data addressing for data access and exchange between the management units. Providing the data interface connecting the communication platform and the energy management platform, allows the latter to access the data. The middleware is supported by the security and privacy mechanisms to protect the exchanged data.

The energy management platform is placed on top of the communication platform. It includes the logic modules or services that perform different kinds of operations based on the data provided by the communication platform and also provide their results and control signals back through the communication platform as well. These services are supported by security and privacy mechanisms that operate at a higher level than their counterparts of the communication platform. Currently we have defined only two main services for energy management, namely, energy balancing and grid resilience, but the general e-balance approach needs not to be limited to these two.

The energy balancing service provides the estimates for energy to be produced and consumed in the near future. This estimation is based on the historic consumption data, but also on real time data and on additional parameters, like the weather forecast data and consumer preferences. This service compares the predicted values with the actual situation and triggers actions on the devices and units under its control to keep its part of the grid in a stable state with respect to energy production and consumption. On the other hand, the grid resilience 
service analyses the state of the grid segment, based on the grid monitoring, and generates control signals to control the grid quality of service.

These two services are responsible for different aspects but they cooperate closely. They provide the status and summary to their counterparts on the parent management unit and generate control signals for the subordinate management units and actuators. These control signals steer the actions necessary for both energy balancing and management.

The communication platform can be regarded as a distributed and secure data exchange platform whereas the energy management platform stands for distributed and combined logic within the grid.

\section{The Communication Platform}

The communication platform provides communications for the different devices in the e-balance system, which consists of a heterogeneous set of devices with different requirements and constraints that communicate using different communication standard protocols. The communication platform is formed mainly by the data storage and exchange middleware that hides this underlying complexity and offers a common interface, called the Data Interface that is used on all the management units in the system by the services providing the energy management functionality. It also deals with issues such as security, privacy and dependability. Figure 2 shows the different modules the communication platform is composed of. In addition, it shows the underlying energy infrastructure the ebalance system interacts with, using the sensors and actors - also a heterogeneous set of hardware devices that support different communication technologies. The middleware comprises standard protocols and communication technologies. As an example, in Figure 2 , three different communication and application protocols or abstractions are depicted, namely DLMS/COSEM [11], IEC 61850 [12] and tinyDSM [13].

The Request Processor module handles all the data accesses, from both the local and the external sources. The Data Access Control grants only accesses that were allowed by the data owner in her security and privacy policy. The Maintenance and Group Management module takes care about discovering and managing the surrounding devices and monitoring changes in that respect. The data is locally stored in the Data Persistence module and all the supported protocols are translated by the Communication Manager module.

\section{The Energy Management Platform}

The energy management platform consists of modules that provide different energy management services. For simplicity, we call them services from now on. Currently there are three services, namely the Energy Balancing service, the Grid Resilience service and the
User Interface service, as depicted in Figure 2. The logic blocks are supported by the security and privacy mechanisms that allow them to access the data interface of the communication platform.

The energy balancing service consists of four major blocks. The main one is the Energy Balancing Logic module. It is responsible for balancing energy in the local part of the grid. The algorithms used in this module are presented in [14]. To achieve this it uses the predicted values for energy consumption and for production, generated by the Consumption Predictor and by the Production Predictor modules. It controls the devices in its area via the Device Controller.

The grid resilience service consists of several modules providing the monitoring of the DER power flows, management of faults in LV and public lighting grid segments, monitoring the LV grid quality, as well as detecting frauds and technical losses in LV, while anticipating LV fault prevention. The DER Power Flows block provides also similar functionality for the MV part of the grid, namely for fault detection and location, comprising self-healing mechanisms. Moreover, it provides MV optimal power flow with validation of optimized solutions, aiming at reducing MV grid losses.

The user interface service is actually an application that runs on the management unit and provides the presentation of the data. The GUI module can also be equipped with advanced functionality for providing key performance indicator statistics or to perform analysis for advising the users on their energy behaviour.

\section{Pilot Demonstrators}

The e-balance system will run in two real-life pilots at the region of Batalha, Portugal and in the holiday park Bronsbergen, the Netherlands [15].

The main focus of the Batalha pilot is on demonstrating the use cases concerned with new functionalities from the point of view of the DSO, including grid monitoring and improvement of grid reliability. In this case, the deployment is done in the distribution grid of EDP Distribuição.

In the case of the Bronsbergen pilot, the focus is on demonstrating use cases concerned with energy balancing, including the interaction with the end users. The deployment is done in the distribution grid of Alliander and in the client homes.

Within the e-balance project, management units, following the described architecture, have been designed and implemented for the different levels of the grid, from the home level up to the MV level. They are being integrated and deployed in the pilot networks. A final phase of the project will consist in the practical evaluation of results in the pilot networks. 
All the use cases to be demonstrated have been defined in detail in [16] and altogether they will show the potential of the e-balance system towards energy balancing, grid monitoring and improvement of grid resilience.

\section{Conclusion}

Energy management systems have a key role in the Smart Grid supporting the increasing penetration of distributed energy resources. They are crucial to manage the dynamic conditions in the future energy grids. Without smart energy management systems the energy grid can operate in an unstable and inefficient way.

In this paper, we have introduced our proposal architecture for such an energy management system. Its main characteristic is being hierarchical and fractal-like. In this way, we trust we can improve the flexibility and scalability of such a control system by, e.g., reusing of programming code for the control algorithms at different levels of the grid.

The e-balance system consists of two platforms: the communication platform and the energy management platform, linked via the data interface. The used communication protocols are standard and the secure middleware provides a defined common interface for the different energy management services.

Implementations of the system are being deployed in two real-life pilots, which will demonstrate use cases on grid balancing, grid monitoring and grid reliability, already defined in detail within the project.

\section{Acknowledgements}

This work has been partially funded by the project ebalance (project number: 609132), within the European Commission 7th Framework Programme (FP7SMARTCITIES-2013) and by national funds through Fundação para a Ciência e a Tecnologia (FCT) with reference UID/CEC/50021/2013.

\section{References}

[1] Siano P.: 'Demand response and smart grids-a survey', Renewable and Sustainable Energy Reviews, 2014, vol. 30, pp. 461-478

[2] 'Balancing energy production and consumption in energy efficient smart neighbourhoods, e-balance project site', http://www.e-balanceproject.eu/index.html, accessed 18 June 2016

[3] Gerards M., Jongerden M., et al:: 'High Level System Architecture Specification', Public deliverable D3.1 of e-balance project, FP7-Smartcities-2013, Project number 609132, 2014
[4] Almeida J., et al.: 'Selection of representative use cases', Public deliverable D2.1 of e-balance project, FP7-Smartcities-2013, Project number 609132, 2014

[5] Molderink A., Bakker V., Bosman M. G. C., Hurink J. L., and Smit G. J. M.: 'Management and control of domestic smart grid technology', IEEE Transactions on Smart Grid, 2010, vol. 1, no. 2, pp. 109-119

[6] CEN-CENELEC-ETSI Smart Grid Coordination Group: 'Smart Grid Reference Architecture',

ftp://ftp.cencenelec.eu/EN/EuropeanStandardization/Hot Topics/SmartGrids/Reference_Architecture final.pdf, accessed 18 June 2016

[7] SmartC2Net consortium: 'Revised Architecture \& Use Cases', Public deliverable D2.1, http://www.smartc2net.eu/public-deliverables.html, accessed 18 June 2016

[8] C-Dax consortium: 'Specification of the Initial C-DAX Architecture and Basic Mechanisms, Protocols and Algorithms', Public deliverable D3.1, http://www.cdax.eu/sites/default/files/C-DAX-D3.1.pdf, accessed 18 June 2016

[9] Kim Y., Keqiang H., Thottan M., Deshpande J.: 'Virtualized and Self-Configurable Utility Communication Enabled by Software-Defined Networks', IEEE Smart Grid Communications 2014 proceedings, pp. 422-427

[10] Nunes M., Grilo A., Pinto C., Melo F., Casaca A., Bernardo A., Silva N.: 'Leveraging Fault Detection and Voltage Control in Low Voltage Grids based on Distributed Monitoring', Proceedings of the 22nd International Conference and Exhibition on Electricity Distribution (CIRED 2014), ISSN 2032-9628, June 2014

[11] DLMS User Association home page, http://www.dlms.com, accessed 18 June 2016

[12] International Electrotechnical Commission, Core IEC Standards, http://www.iec.ch/smartgrid/standards/, accessed 16 June 2016

[13] Piotrowski K., Langendoerfer P., Peter St.: 'tinyDSM: A highly reliable cooperative data storage for Wireless Sensor Networks', International Symposium on Collaborative Technologies and Systems, 2009

[14] Gerards M. E. T., Toersche H. A., Hoogsteen G., van der Klauw T., Hurink J. L., Smit G. J. M.: 'Demand side management using profile steering', Powertech 2015, Eindhoven, the Netherlands

[15] Geers M., et al.: 'Specification of the Demonstrators', Public deliverable D6.1 of e-balance 
project, FP7-Smartcities-2013, Project number 609132, 2015.

[16] Peralta J., Jiménez N., Casaca A., Piotrowski K., Melo F., 'Innovative methodology to define stakeholders' requirements for smart systems', Proceedings of the 23rd International Conference and Exhibition on Electricity Distribution (CIRED 2015), ISSN 2032-9644, Lyon, France, June 2015 